\title{
Modification of the Structure and Magnetic Properties of Cobalt-Doped Ferrihydrite Nanoparticles under Heat Treatment
}

\author{
S. V. Stolyar ${ }^{1,2}$, D. A. Balaev ${ }^{1,2}$, A. A. Krasikov², A. A. Dubrovskiy², R. N. Yaroslavtsevev,2, \\ O. A. Bayukov'². N. Volochaev².R. S. Iskhakov² \\ ${ }^{1}$ Siberian Federal University, Krasnoyarsk, Russia \\ ${ }^{2}$ Kirensky Institute of Physics, Federal Research Center KSC SB RAS, Krasnoyarsk, Russia
}

\begin{abstract}
Nanoparticles of antiferromagnetic materials acquire the magnetic moment due to the surface effects and structural defects. According to the Neel hypothesis, magnetic moment $\mu_{\mathrm{P}}$ of a particle containing $N$ magnetically active atoms with magnetic moment $J$ can be estimated as $\mu_{\mathrm{P}} \sim J \cdot N^{n}$ or $\mu_{\mathrm{P}} \sim V^{n}$, where $V$ is the particle volume. Numerous studies of the magnetic properties of ferrihydrite $5 \mathrm{Fe}_{2} \mathrm{O}_{3} \cdot 9 \mathrm{H}_{2} \mathrm{O}$ and ferritin revealed a value of $n \approx 1 / 2$ for this material, in which Fe atoms have the octahedral surrounding of anions. We investigate the effect of low-temperature annealing of cobalt-doped ferrihydrite nanoparticles on their average size and magnetic properties. Using the Mössbauer spectroscopy study, we demonstrate that doping with Co makes $\mathrm{Fe}$ atoms enter the anion tetrahedra, which leads to an increase in the exponent $n>1 / 2$ in the expression $\mu_{\mathrm{P}} \sim J \cdot N^{n}$.
\end{abstract}

\section{Introduction}

At present, fine iron oxide, hydroxide, and oxyhydroxide powders consisting of particles smaller than $10 \mathrm{~nm}$ have been objects of enhanced interest of researchers due to wide application of these powders in catalysis, biomedicine, waste recovery, water purifying, etc. [1-3].

Highly dispersed metastable ferrihydrite is an iron oxyhydroxide that deserves the most attention. Its properties are determined by the composition, structure, and fabrication technique used [4]. The chemical formula of ferrihydrite is usually written in the form $5 \mathrm{Fe}_{2} \mathrm{O}_{3} \cdot 9 \mathrm{H}_{2} \mathrm{O}$, but the number of $\mathrm{OH}$ bonds in the structure can change depending on its defectness.

According to the results reported in $[5,6]$, the ferrihydrite structure represents a sequence of ABAC anion planes with a large number of stacking faults. In the cubic packing element with a sequence of $\mathrm{ABCABC}$ anion planes, $\mathrm{Fe}$ atoms in the octahedral surrounding form two neighboring layers of octahedra occupied by iron. In the hexagonal packing element, where the anion planes are located in the ABAB (ACAC) sequence, single layers of octahedra occupied by iron will form [7]. This structure is confirmed by numerous Mössbauer spectroscopy experiments on nanoparticles of ferrihydrite of different origins and iron-accumulating proteins called ferritins. The Mössbauer measurements detect, as a rule, two nonequivalent octahedral iron ion positions in natural and synthetic ferrihydrites $[7,8]$, the occupancy of which strongly depends of ferrihydrite fabrication methods. 
According to $[9,10]$, the ferrihydrite crystal structure is formed by the ABAC anion packing (R31c, $\mathrm{a} \approx 6 \AA$, and $\mathrm{c} \approx 9 \AA$ ), where iron atoms are located in both the tetrahedral and octahedral voids. As was shown in [11], in the ideal ferrihydrite structure $20 \%$ of iron atoms should be localized in tetrahedra. Thus, the questions about the presence of tetrahedral positions of iron atoms in the ferrihydrite structure and effect of these positions on the magnetic properties of the material remain unanswered.

Ferrihydrite nanoparticles are characterized by the antiferromagnetic (AFM) ordering, but acquire the magnetic moment due to surface effects and structural defects. At room temperature, ferrihydrite nanoparticles are in the superparamagnetic (SP) state. The controlled variation in the size of ferrihydrite nanoparticles, their defectness, and, consequently, magnetic properties is the urgent practical problem. In [12], we prepared ferrihydrite nanoparticle powders doped with cobalt in a ratio of $\mathrm{Fe} / \mathrm{Co}=5 / 1$ by hydrolysis, determined their blocking temperatures, and established the particle size, magnetization, and surface and bulk anisotropy constants. The aim of this work was to investigate the modification of the magnetic properties of synthetic cobalt-doped ferrihydrite nanoparticles under heat treatment.

\section{Experimental}

Cobalt-doped chemical ferrihydrite samples were prepared at room temperature by slow adding the $\mathrm{NaOH}$ alakli solution to the iron chloride $\mathrm{FeCl}_{3}$ and cobalt (III) salt solution at continuous intermixing to attain the neutral $\mathrm{pH}$ value [12]. The precipitate was collected on a filter, washed, and dried at room temperature. Low-temperature annealing was performed in a drying oven at a temperature of $\sim 170^{\circ} \mathrm{C}$ for 3 and $24 \mathrm{~h}$, as was described in $[13,14]$. Hereinafter, the samples are marked in accordance with the annealing time in hours, i.e., $\mathrm{S}-\mathrm{FH}(\mathrm{Co})-\mathrm{Oh}, \mathrm{S}-\mathrm{FH}(\mathrm{Co})-3 \mathrm{~h}$, and $\mathrm{S}$ $\mathrm{FH}(\mathrm{Co})-24 \mathrm{~h}$. Electron microscopy investigations were carried out on a Hitachi HT7700 transmission electron microscope at an accelerating voltage of $100 \mathrm{kV}$ at the Center of Collective Use of the Federal Research Center KSC SB RAS. The elemental distribution in particle aggregates was obtained from energy dispersive spectra (EDS) on a Hitachi TM 3000 microscope. The amount of Co atoms with respect to the amount of $\mathrm{Fe}$ atoms in the samples determined from the obtained EDS spectra was $18 \%$ [12]. Mössbauer spectra were measured on an MC-1104Em spectrometer with the ${ }^{57} \mathrm{Co}(\mathrm{Cr})$ source at room temperature on powder samples with a thickness of $5-10 \mathrm{mg} / \mathrm{cm}^{2}$ on the basis of the natural iron content. Isomer chemical shifts are indicated relative to $\alpha$-Fe. The magnetic measurements were performed on a vibrating sample magnetometer. The investigated powder was fixed in a measuring capsule in paraffin. The magnetic moment data are given in emu per unit mass of the investigated sample. Temperature dependences of the magnetic moment $\mathrm{M}(\mathrm{T})$ were obtained in the zero field cooling (ZFC) and field cooling (FC) modes. 


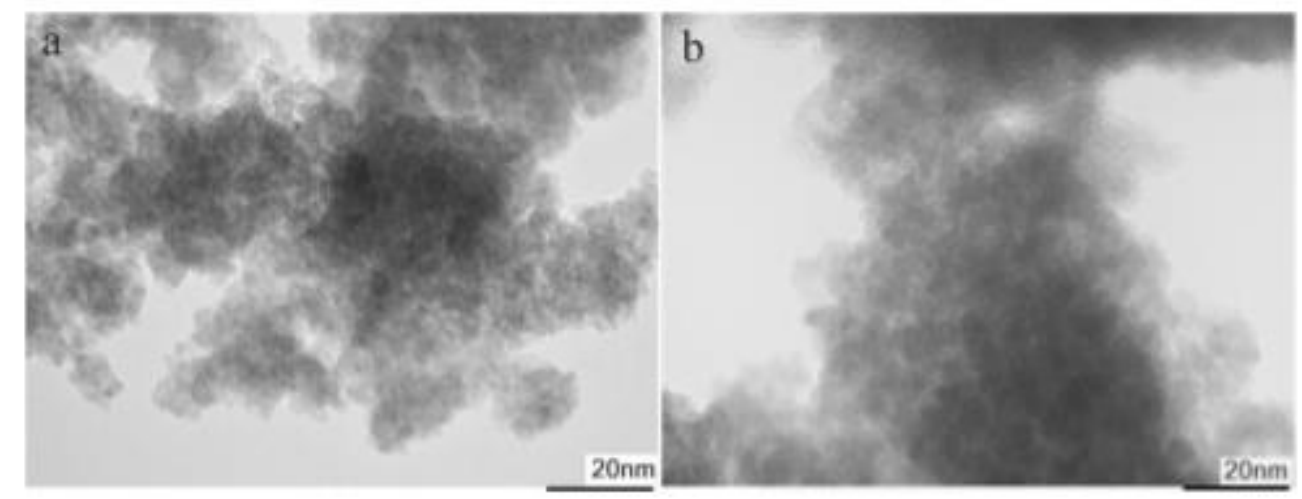

Fig. 1. Transmission electron microscopy images of particles of samples (a) S-FH(Co)-Oh and (b) S$\mathrm{FH}(\mathrm{Co})-24 \mathrm{~h}$.

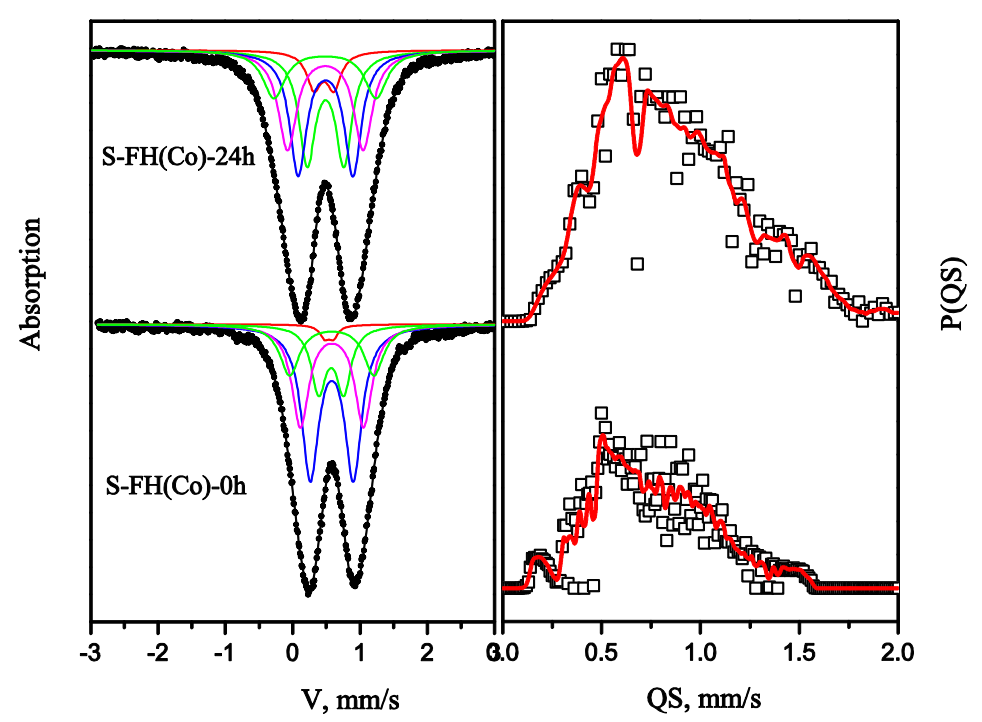

Fig. 2. Mössbauer spectra, their partial components, and quadrupole splitting distribution $P(\mathrm{QS})$ for the investigated samples.

\section{Results}

\subsection{Electron microscopy and Mössbauer spectroscopy study}

Figure 1 shows transmission electron microscopy (TEM) images of samples S-FH(Co)-Oh and $\mathrm{S}-\mathrm{FH}(\mathrm{Co})-24 \mathrm{~h}$. The average particle sizes were found to be $\sim 3.5$ and $\sim 4.9 \mathrm{~nm}$ for samples $\mathrm{FH}(\mathrm{Co})-0 \mathrm{~h}$ and S-FH(Co)-24h, respectively. Figure 2a shows room-temperature Mössbauer spectra of samples S$\mathrm{FH}(\mathrm{Co})-0 \mathrm{~h}$ and $\mathrm{S}-\mathrm{FH}(\mathrm{Co})-24 \mathrm{~h}$. The spectra represent quadrupole doublets characteristic of unblocked SP particles with different line broadenings. Analysis of the quadrupole splitting distribution $P(\mathrm{QS})$ in the experimental spectra (Fig. 2b) suggests the presence of several nonequivalent iron positions with 
different degrees of distortion of the local surrounding. The model spectra were formed with regard to the specific features of the $P(\mathrm{QS})$ distribution and fit to the experimental spectra upon variation in the entire set of superfine parameters. The results of Mössbauer spectra identification are given in Table 1.

Table 1. Mössbauer parameters of ferrihydrite. IS is the isomer shift, QS is the quadrupole splitting, W is the linewidth, and $\mathrm{A}$ is the position occupancy. Positions $\mathrm{Fe} 1$ and $\mathrm{Fe} 2$ correspond to the cubic and hexagonal ligand packing, respectively, and Fe3, to the interlayer iron atoms.

\begin{tabular}{|c|c|c|c|c|c|}
\hline Sample & $\begin{array}{l}\mathrm{IS}, \mathrm{mm} / \mathrm{s} \\
\quad \pm 0.05\end{array}$ & $\begin{array}{l}\mathrm{QS}, \mathrm{mm} / \mathrm{s} \\
\pm 0.02\end{array}$ & $\begin{array}{c}\mathrm{W}, \mathrm{mm} / \mathrm{s} \\
\quad \pm 0.02\end{array}$ & $\mathrm{~A}, \pm 0.03$ & Position \\
\hline \multirow[t]{5}{*}{$\mathrm{S}-\mathrm{FH}(\mathrm{Co})-\mathrm{Oh}$} & 0.296 & 0.12 & 0.15 & 0.01 & Fe1(4)-cub \\
\hline & 0.333 & 0.36 & 0.27 & 0.15 & $\mathrm{Fe} 1(6)$-cub-sym \\
\hline & 0.345 & 0.63 & 0.31 & 0.40 & Fe1(6)-cub-dis \\
\hline & 0.347 & 0.94 & 0.32 & 0.30 & Fe2(6)-hex \\
\hline & 0.343 & 1.26 & 0.34 & 0.14 & Fe3(6)-inter \\
\hline \multirow[t]{5}{*}{ S-FH(Co)-24h. } & 0.309 & 0.29 & 0.26 & 0.08 & Fe1(4)-cub \\
\hline & 0.334 & 0.54 & 0.29 & 0.23 & Fe1(6)-cub-sym \\
\hline & 0.333 & 0.81 & 0.31 & 0.29 & Fe1(6)-cub-dis \\
\hline & 0.329 & 1.12 & 0.33 & 0.25 & Fe2(6)-hex \\
\hline & 0.325 & 1.51 & 0.39 & 0.15 & Fe3(6)-inter \\
\hline
\end{tabular}

\subsection{Magnetic properties}

Figure 3 shows FC and ZFC temperature dependences of the magnetic moment for samples S$\mathrm{FH}(\mathrm{Co})-\mathrm{Xh}$ annealed for different periods of time. The M(T) dependences are typical of SP systems: one can see the maximum at temperature $T_{\mathrm{B}}$ under the ZFC conditions and effect of the thermomagnetic prehistory at temperatures below $T_{\mathrm{B}}$. It can be seen that the annealing leads to a significant increase in the $T_{\mathrm{B}}$ value. Figure 4 presents magnetic hysteresis loops at $T=4.2 \mathrm{~K}$ for samples $\mathrm{S}-\mathrm{FH}(\mathrm{Co})-0 \mathrm{~h}$ and $\mathrm{S}-\mathrm{FH}(\mathrm{Co})-24 \mathrm{~h}$. It can be seen that coercivity $H_{C}$ of the investigated 
ferrihydrite powders grows after heat treatment $\left(H_{C} \approx 5.15 \mathrm{kOe}\right.$ for $\mathrm{S}-\mathrm{FH}(\mathrm{Co})-0 \mathrm{~h}$ and $H_{C} \approx 8.8 \mathrm{kOe}$ for S-FH(Co)-24h).

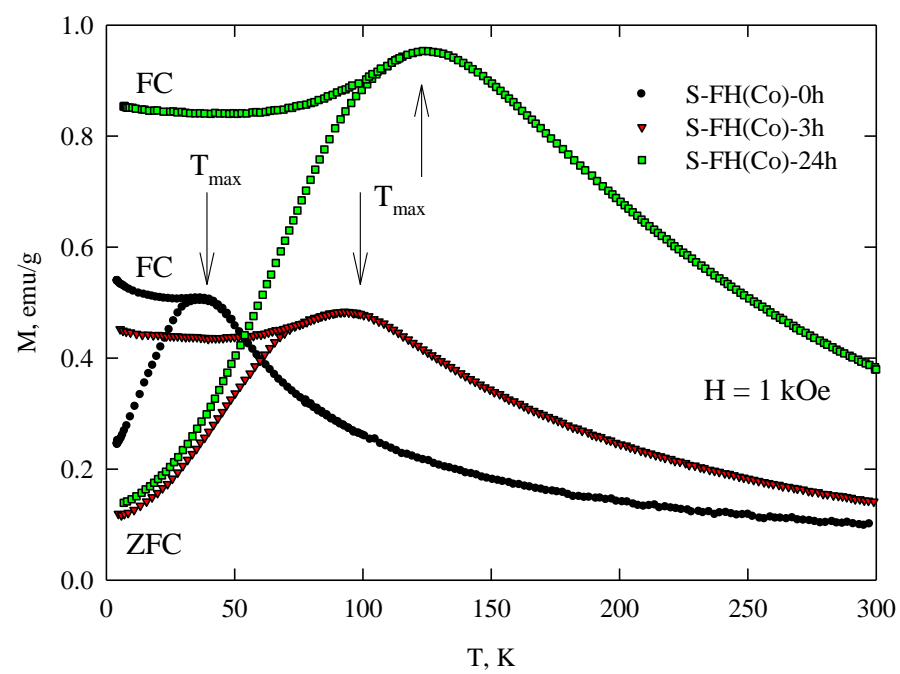

Fig. 3. Temperature dependences of the magnetic moment for the investigated chemical ferrihydrite samples.

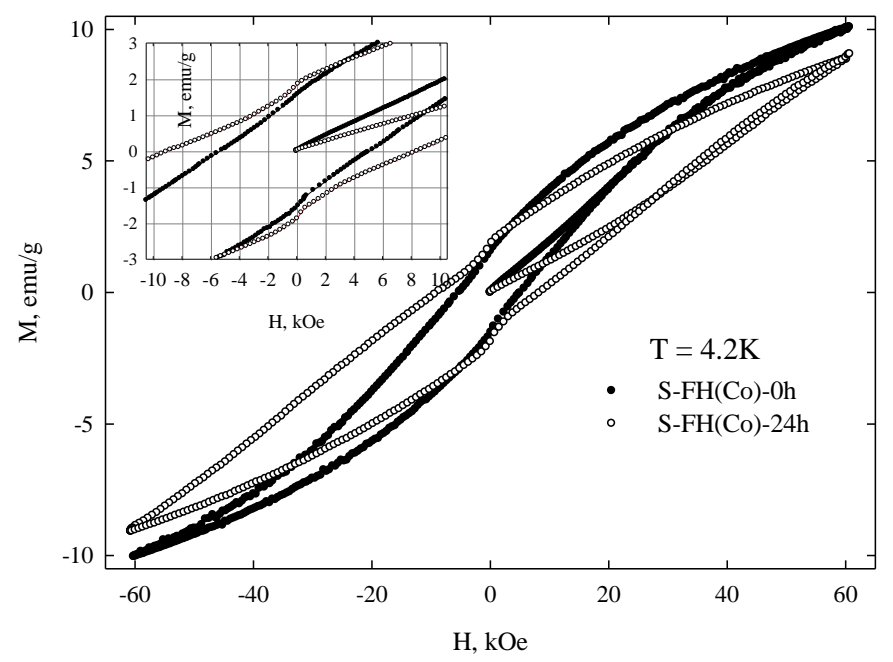

Fig. 4. Magnetic hysteresis loops for samples $\mathrm{S}-\mathrm{FH}(\mathrm{Co})-0 \mathrm{~h}$ and $\mathrm{S}-\mathrm{FH}(\mathrm{Co})-24 \mathrm{~h}$ at $T=4.2 \mathrm{~K}$. Inset: enlarged weak-field range.

\section{Discussion}

The main difference between the behaviors of nanosized AFM particles and their bulk analogs is the occurrence of the uncompensated magnetic moment [15-20]. According to the Neel hypothesis $[21,22]$, the magnetic moment $\mu_{\mathrm{P}}$ of a particle containing $N$ magnetically active atoms with magnetic moment $J$ can be estimated using the expression $\mu_{\mathrm{P}} \sim J \cdot N^{n}$ or

$$
\mu_{\mathrm{P}} \sim V^{n}
$$


since $N \sim V$ ( $V$ is the particle volume). The exponent $n$ for a particle with the odd number of ferromagnetic planes is $2 / 3$. Random magnetic order breaks caused by structural defects lead to decompensation of the AFM-ordered spins; in this case, the $n$ value can take the values between $\sim(1 / 3-1 / 2)[21,22]$. The uncompensated magnetic moment of particles starts playing an important role in the magnetic behavior of AFM particles when the number of atoms in a particle is about $10^{3}-$ $10^{4}[20,23]$. Numerous investigations of ferrihydrite and ferritin reliably demonstrated that nanoparticles of this material are characterized by an exponent of $n \approx 1 / 2$ [13-17, 24-28].

We start discussing the experimental data with the Mössbauer spectra. The ideal ferrihydrite structure contains alternating doubled and single layers of octahedral iron positions along the $c$ axis. The doubled layers are characterized by the $\mathrm{ABC}$ cubic structure and the single layers, by the hexagonal packing of $\mathrm{ABAB}$ ligands. Consequently, the Mössbauer spectra of the real ferrihydrites contain quadrupole doublets corresponding to the cubic Fe1 and hexagonal Fe2 ligand packings, as well as $\mathrm{Fe} 3$ positions attributed to the interlayer iron [7, 12, 13, 29]. The spectra of investigated cobalt-doped ferrihydrite contain two sorts of octahedral positions in the doubled iron layers, Fe1(6)cub-sym and Fe1(6)-cub-dis, with different degrees of distortion of the coordination octahedron. In addition, we found the Fe1(4)-cub doublets with the small chemical shift and small quadrupole splitting, which are typical of the tetrahedral coordination over ligands. The presence of the tetrahedral positions is characteristic of the cubic packing of ligands. The occupancy of such positions in the initial S-FH(Co)-0h sample is small and attains $8 \%$ in sample $\mathrm{S}-\mathrm{FH}(\mathrm{Co})-24 \mathrm{~h}$. Cobalt atoms entering the cubic phase occupy the octahedral positions and replace iron to the tetrahedra. It should be noted that the occupancy of the $\mathrm{Fe} 3$ (defect) position, which characterizes the interlayer atoms, remains invariable after heat treatment. Summarizing the results of the Mössbauer spectra analysis, we may conclude that cobalt enters the ferrihydrite structure and the annealing does not lead to the occurrence of new crystallographic phases; however, defectness of the crystal structure increases due to an increase in the number of tetrahedral positions of iron atoms.

The observed $M(T)_{\text {ZFC }}$ maximum (Fig. 3) is undoubtedly related to the SP blocking temperature $T_{\mathrm{B}}$ of particles ${ }^{1}$. The $T_{\mathrm{B}}$ value, in turn, is related to particle volume $V$ and effective magnetic anisotropy constant $K_{\text {eff }}$ in accordance with the classical Néel-Brown relation, which can be written for the case of quasi-static magnetic measurements as

$$
T_{\mathrm{B}} \approx K_{\text {eff }} V / 25 k_{\mathrm{B}}
$$

\footnotetext{
${ }^{1}$ The average particle size corresponds to certain average blocking temperature $\left\langle T_{\mathrm{B}}\right\rangle$ related to temperature $T_{\max }$ observed in the $M(T)_{\mathrm{ZFC}}$ dependence via the proportionality coefficient depending on the distribution type [25-28] and exponent $n$ in expression (1) (at $n=1$, expression (1) describes ferroor ferrimagnetic particles).
} 
where $k_{\mathrm{B}}$ is the Boltzmann constant. Taking into account expression (2), we may conclude that the $T_{\mathrm{B}}$ growth after annealing (see Fig. 3) reflects the growth in the particle size, which is consistent with the TEM data (see Fig. 2 and Section 3.1). Since we have $V \sim d^{3}$, then, with regard to expression (2), under the assumption about the retained effective anisotropy constant of the investigated nanoparticles after heat treatment, the initial "1" and annealed "2" ferrihydrite powders should meet the relations $V_{2} / V_{1} \approx d_{2}{ }^{3} / d_{1}{ }^{3} \approx T_{\mathrm{B} 2} / T_{\mathrm{B} 1}$. Indeed, for ferrihydrite samples $\mathrm{S}-\mathrm{FH}(\mathrm{Co})-0 \mathrm{~h}$ and $\mathrm{S}-\mathrm{FH}(\mathrm{Co})-24 \mathrm{~h}$ we have $d_{2}{ }^{3} / d_{1}{ }^{3} \approx 2.9$, whereas $T_{\mathrm{B} 2} / T_{\mathrm{B} 1} \approx 3.4$. It is reasonable to explain the particle coarsening upon annealing by agglomeration of closely adjacent particles $[13,14]$.

In addition, the increase in the particle size is indirectly confirmed by the growth of coercivity $H_{C}$ of the samples at temperatures below $T_{\mathrm{B}}$. The magnetic hysteresis loops obtained for the investigated powders at $T=4.2 \mathrm{~K}$ (Fig. 4) are indicative of the coercivity growth by a factor of 1.7 after heat treatment. Coercivity $H_{C}$ of single-domain particles with saturation magnetization $M_{S}$ is determined using the expression following from the Stoner-Wohlfarth model [30]

$$
H_{C} \approx\left(K_{\text {eff }} / M_{S}\right)\left\{1-\left(T / T_{B}\right)^{0.5}\right\}
$$

All the parameters in the right-hand side of expression (3) can depend on the particle size. We only consider the effect of $M_{S}$ on $H_{C}$ in AFM particles. Since $M_{\mathrm{S}}=\mu_{\mathrm{P}} / V$, from expression (1) we obtain $M_{S} \sim V^{n-1}$. Consequently, from (3) we have $H_{C} \sim V^{1-n}$ and, since the $n$ value lies between $1 / 3 \leq n \leq 2 / 3$ (Section 1), then the increase in the particle size should lead to the coercivity growth, which was observed in the experiment.

Figure 5 summarizes the effect of annealing time on blocking temperature $T_{\mathrm{B}}$ and particle size $d$ determined from the TEM data (Section 3.1) for the synthesized ferrihydrite powder (left axis). The blocking temperature and particle size increase after low-temperature annealing. According to the data illustrated in Fig. 3, the room-temperature magnetic moment also increases after annealing. Figure 5 (right axis) presents also the values $\chi(300 \mathrm{~K})=M(300 \mathrm{~K}) / H$ vs annealing time for the synthesized ferrihydrite powder. However, the observed increase in the $\chi(300 \mathrm{~K})$ values of the annealed ferrihydrite powders, i. e., the increase in the particle size is already difficult to explain using relations (1) and (2). Indeed, the magnetic susceptibility of an ensemble of AFM nanoparticles is determined by the SP response $\chi_{\mathrm{SP}}$ of the system and AFM susceptibility $\chi_{\mathrm{AF}}$ of the antiferromagnet [15]: $\chi=\chi_{\mathrm{SP}}+\chi_{\mathrm{AF}}$ (usually, $\left.\chi_{\mathrm{SP}}>\chi_{\mathrm{AF}}\right)[25]$. In the unblocked state $\left(T>T_{\mathrm{B}}\right)$, in weak fields (or at high temperatures), we have $\chi_{\mathrm{SP}}=N_{\mathrm{P}}<\mu_{\mathrm{P}}>^{2} / 3 k_{\mathrm{B}} T$, where $N_{\mathrm{P}}$ is the number of particles per unit sample mass and $\left\langle\mu_{\mathrm{P}}\right\rangle$ is the average magnetic moment of a particle [15]. Based on expression (1), we can obtain the functional dependence of $\chi_{\text {SP }}$ on the particle volume (at $T=$ const):

$$
\chi_{\mathrm{SP}}(V) \sim N_{\mathrm{P}} V^{2 n}
$$




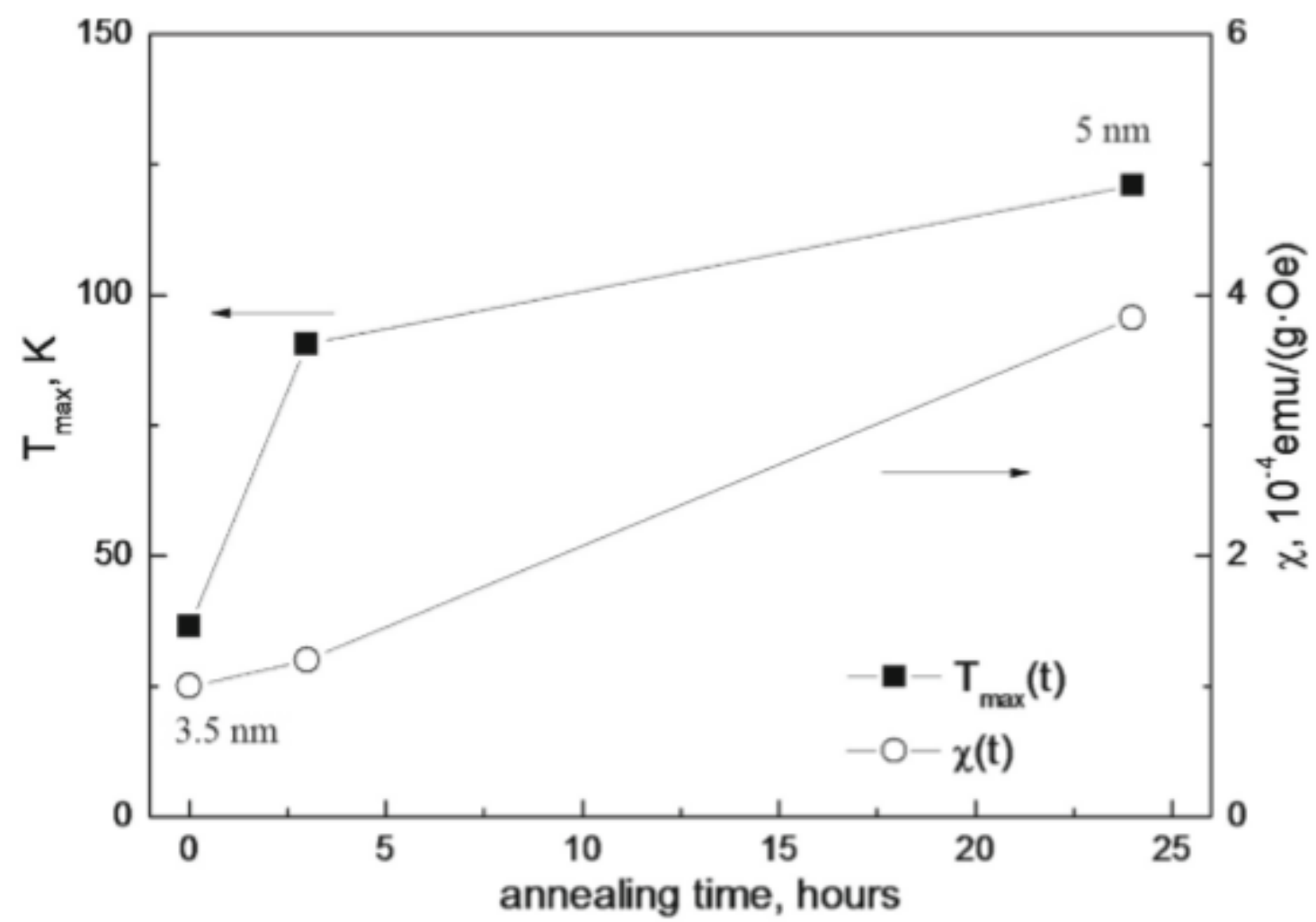

Fig. 5. Blocking temperature $T_{\mathrm{B}}$ and magnetic susceptibility $\chi(\mathrm{T}=300 \mathrm{~K})$ of ferrihydrite vs annealing time. Particle sizes determined from the TEM data for the initial samples and the samples annealed for $24 \mathrm{~h}$ are shown.

For an ideal system of nanoparticles with the variable size, it is obvious that $N_{\mathrm{P}} V=$ const; as a result, $N_{\mathrm{P}} \sim 1 / V$. Consequently, for such an ideal system, we have $\chi_{\operatorname{SP}}(V) \sim(1 / V) V^{2 n} \sim V^{2 n-1}$. Using the data of numerous studies on ferrihydrite and ferritin, it was reliably established that the exponent for nanoparticles of this material is $n \approx 1 / 2[13-17,24-28]$ and, in this case, the susceptibility of an ideal system of ferrihydrite nanoparticles should not depend on the particle size. However, the increase in the ferrihydrite particle size upon annealing was proven by both the direct (TEM data) and indirect (increasing $T_{\mathrm{B}}, H_{\mathrm{C}}$, and $\mu_{\mathrm{P}}$ [13]) measurements and with an increase in the particle volume by a factor of about 3 , the $\chi(300 \mathrm{~K})$ value increase by a factor of almost 4 . The relatively weak magnetic susceptibility growth with increasing particle volume $V$ is possible only with an increase in the exponent $n>1 / 2$. In our opinion, the latter is caused by the occurrence of additional planes in the ferrihydrite structure, which are formed by the tetrahedral positions of iron atoms in the Co-doped powders investigated by us. 


\section{Conclusions}

We experimentally confirmed the possibility of purposeful modification of the size of synthetic ferrihydrite nanoparticles and their defectness at sufficiently low $\left(\sim 170^{\circ} \mathrm{C}\right)$ temperatures. According to the data on cobalt-doped ferrihydrite, annealing for $24 \mathrm{~h}$ significantly increases the superparamagnetic blocking temperature and multiply enhances the particle volume and magnetic susceptibility, which is accompanied by the growth in the number of tetrahedral positions of iron atoms in the ferrihydrite structure. Exposure of the investigated cobalt-doped ferrihydrite powders in saturated water vapors or aqueous medium already does not affect the size and magnetic characteristics of particles.

\section{References}

[1] A. A. Khassin, A. G. Sipatrov, M. P. Demeshkina, and T. P. Minyukova, React. Kinet. Catal. Lett. 97, 371 (2009).

[2] J. A. Dyer, P. Trivedi, S. J. Sanders, N. C. Scrivner, and D. L. Sparks, J. Colloid Interface Sci. 270, 66 (2004).

[3] K. Dobretsov, S. Stolyar, and A. Lopatin, Acta Otorhinolaryngol. Ital. Organo Uff. Della Soc. Ital. Di Otorinolaringol. E Chir. Cerv.-Facc. 35, 97 (2015).

[4] E. Murad and U. Schwertmann, Am. Mineral. 65, 1044 (1980).

[5] E. Jansen, A. Kyek, W. Schafer, and U. Schwertmann, Appl. Phys. A Mater. Sci. Process. 74, s1004 (2002).

[6] F. V. Chukhrov, B. B. Zvyagin, and A. I. Gorshkov, Izv. Akad. Nauk SSSR, Ser. Geol. 4, 23 (1973).

[7] S. V. Stolyar, O. A. Bayukov, Y. L. Gurevich, V. P. Ladygina, R. S. Iskhakov, and P. P. Pustoshilov, Inorg. Mater. 43, 638 (2007).

[8] S. V. Stolyar, O. A. Bayukov, Y. L. Gurevich, R. S. Ishkakov, and V. P. Ladygina, Bull. Russ. Acad. Sci. Phys. 71, 1286 (2007).

[9] V. A. Drits, Clay Miner. 28, 185 (1993).

[10] D. A. Yatsenko, V. P. Pakharukova, S. V. Tsybulya, A. A. Matvienko, and A. A. Sidel'nikov, J. Struct. Chem. 53, 548 (2012).

[11] F. M. Michel, L. Ehm, S. M. Antao, P. L. Lee, P. J. Chupas, G. Liu, D. R. Strongin, M. A. A. Schoonen, B. L. Phillips, and J. B. Parise, Science (80-. ). 316, 1726 (2007).

[12] S. V. Stolyar, R. N. Yaroslavtsev, R. S. Iskhakov, O. A. Bayukov, D. A. Balaev, A. A. 
Dubrovskii, A. A. Krasikov, V. P. Ladygina, A. M. Vorotynov, and M. N. Volochaev, Phys. Solid State 59, 555 (2017).

[13] D. A. Balaev, A. A. Krasikov, A. A. Dubrovskiy, S. I. Popkov, S. V. Stolyar, O. A. Bayukov, R. S. Iskhakov, V. P. Ladygina, and R. N. Yaroslavtsev, J. Magn. Magn. Mater. 410, 171 (2016).

[14] D. A. Balaev, A. A. Krasikov, A. A. Dubrovskii, S. V. Semenov, O. A. Bayukov, S. V. Stolyar, R. S. Iskhakov, V. P. Ladygina, and L. A. Ishchenko, J. Exp. Theor. Phys. 119, 479 (2014).

[15] S. Mørup, D. E. Madsen, C. Frandsen, C. R. H. Bahl, and M. F. Hansen, J. Phys. Condens. Matter 19, 213202 (2007).

[16] S. A. Makhlouf, F. T. Parker, and A. E. Berkowitz, Phys. Rev. B 55, R14717 (1997).

[17] A. Punnoose, T. Phanthavady, M. S. Seehra, N. Shah, and G. P. Huffman, Phys. Rev. B 69, 054425 (2004).

[18] A. Punnoose, H. Magnone, M. S. Seehra, and J. Bonevich, Phys. Rev. B 64, 174420 (2001).

[19] S. A. Makhlouf, H. Al-Attar, and R. H. Kodama, Solid State Commun. 145, 1 (2008).

[20] Y. L. Raikher and V. I. Stepanov, J. Exp. Theor. Phys. 107, 435 (2008).

[21] L. Néel and C. R. Acad, Sci. Paris 253, 1286 (1961).

[22] L. Néel and C. R. Acad, Sci. Paris 253, 203 (1961).

[23] Y. L. Raikher and V. I. Stepanov, J. Phys. Condens. Matter 20, 204120 (2008).

[24] D. A. Balaev, A. A. Dubrovskii, A. A. Krasikov, S. V. Stolyar, R. S. Iskhakov, V. P. Ladygina, and E. D. Khilazheva, JETP Lett. 98, 139 (2013).

[25] N. J. O. Silva, V. S. Amaral, and L. D. Carlos, Phys. Rev. B 71, 184408 (2005).

[26] C. Gilles, P. Bonville, H. Rakoto, J. M. Broto, K. K. W. Wong, and S. Mann, J. Magn. Magn. Mater. 241, 430 (2002).

[27] N. J. O. Silva, A. Millán, F. Palacio, E. Kampert, U. Zeitler, H. Rakoto, and V. S. Amaral, Phys. Rev. B 79, 104405 (2009).

[28] J. G. E. Harris, J. E. Grimaldi, D. D. Awschalom, A. Chiolero, and D. Loss, Phys. Rev. B 60, 3453 (1999).

[29] S. V. Stolyar, O. A. Bayukov, V. P. Ladygina, R. S. Iskhakov, L. A. Ishchenko, V. Y. Yakovchuk, K. G. Dobretsov, A. I. Pozdnyakov, and O. E. Piksina, Phys. Solid State 53, 100 (2011). 
[30] E. C. Stoner and E. P. Wohlfarth, Philos. Trans. R. Soc. A Math. Phys. Eng. Sci. 240, 599 (1948). 